Martín-Pastor, Andrés \& Narváez Rodríguez, Roberto. New properties about the intersection of rotational quadratic surfaces and their applications in architecture. Nexus Network Journal Vol. 21, no.1 (2019). pp. 175-196 https://doi.org/10.1007/s00004-018-0420-x

\title{
NEW PROPERTIES ABOUT THE INTERSECTION OF ROTATIONAL QUADRATIC SURFACES AND THEIR APPLICATIONS IN ARCHITECTURE
}

Andrés Martín-Pastor. ETSIE. Dpto. de Ingeniería Gráfica. Universidad de Sevilla. Email: archiamp@us.es

Roberto Narváez Rodríguez. ETSIE. Dpto. de Ingeniería Gráfica. Universidad de Sevilla

Keywords: Quadric Theorems, Descriptive Geometry, Treatises, Architectural Geometry, Confocal Surfaces, Rotational Quadratic Surfaces, Graphic Thinking

\begin{abstract}
A graphic conjecture is presented based on a singular property stated by Archimedes [287-212 B.C.] in his work On Conoids and Spheroids. This ancient text constitutes the starting argument for graphic research that has revealed an unknown property regarding the intersection of rotational quadratic surfaces which they share one of their foci. This article shows the heuristicgeometric reasoning carried out stemming from Archimedes' text transcriptions and a conjecture that can be deduced when the initial property is generalised for the rest of the quadratic surfaces. Moreover, an explanation is offered for the possibilities of this property to be used for the discretisation of architectural surfaces through the use of parametric design and digital fabrication.
\end{abstract}

The property discovered in this research is summarised as follows: "If two rotational quadratic surfaces share the position of one of their foci at the same point, then the intersection curves between the two surfaces are always planar". ${ }^{1}$

This new property, which currently remains only a conjecture ${ }^{2}$, has been formulated from purely graphic thinking. However, its validity has been fully tested through a heuristic method which involves checking the planarity on all possible combinations of quadric intersections in a necessary and sufficient number of cases. For this purpose, the power of CAD tools has been used as a true geometric research laboratory where the validity of the theoretical approaches is subject to trial and error.

\footnotetext{
${ }^{1}$ The oblate ellipsoid and one-sheeted hyperboloid are excluded.

2 The academic community cannot consider it a 'theorem' because it has yet to be proven in a formal mathematical language.
} 


\section{Introduction}

Graphic sketches have always been linked to Mankind's way of reasoning. Mathematical-logic abstraction is a human skill that interweaves with the innate capacity of expressing symbols and concepts with graphics. However, it must be borne in mind that the great majority of illustrations of the Greek and Latin texts were created during the Renaissance period by means of several translations and transcriptions of the original surviving texts. The Renaissance opened new possibilities for coded graphical language with a new visual and procedural configuration that would continue to be refined during the XVII century. The art-science merger from that period, and the proliferation of specific treatises in collaboration with printing, also helped towards the development of a graphic tool that was useful for mathematicians, painters, and architects alike.

The multiview parallel projection system, already used by Piero della Francesca [c.1412-1492] and Albrecht Dürer [1471-1528], and the codification of central projection, and perspective, proposed by Filippo Brunelleschi [1377-1446] remained practically untouched until the early XIX century. The codification of perspective was slow, and although the conceptual basis had already been established in the XV century, issues of significant controversy arose in the XVI and XVII centuries. For such codification, the contribution by great mathematicians is undeniable, among whom Federico Comandino [1509-1575] and Guidobaldo Burbon del Monte [1545-1607] deserve special mention. Commadino's work was graphically interpreted and translated by Daniele Barbaro [1513-1570]. Guidobaldo's work was eventually interpreted by Jean François Niçeron [1613-1646], who carried out the objective representation of the shadow cast by the sun. With this contribution, the definition of perspective was concluded, almost fifty years after the problem had been proposed by Guidobaldo. Girard Desargues [1591-1661] also contributed with the first theorems regarding projective geometry and his speculations about infinity within the perspective system itself. ${ }^{3}$

It can be appreciated that the history of graphic thinking is full of complexities and contradictions and that it neither follows a linear course nor is it associated in parallel with rigorously mathematical thinking in all cases (Raynaud, 2018:245). Gaspard Monge [1746-1818], a professor in the École Polytechnique of Paris, combines the previous graphical thinking Géométrie Descriptive (1798) with the most innovative mathematical development of the time applied to the study of surfaces: Application de l'Analyse à la Géométrie (1809). Several of the most well-known theorems regarding the intersection of quadratic surfaces are due to Monge and to many of his successors, as shown in the following paragraphs.

Since the appearance of Monge's work, the discipline of Descriptive Geometry -Geometry from graphics- has held special prominence in engineering degrees, and later in that of architecture. From that moment onwards, the importance of graphic thinking would remain consolidated as a structural aspect for the training in these disciplines and Descriptive Geometry would be present in the syllabi of the most prestigious schools.

Currently, in the post digital era in which we live, CAD tools are no longer considered 'new technology', but as everyday instruments. We refer to 'graphic thinking' as a way of reasoning in which the construction of the drawing takes an active part in the development of the reasoning. This is performed in such a way that the internal coherence of the graphic

\footnotetext{
${ }^{3}$ For in-depth information regarding the mathematical controversies on the representation of the solar shadow in 17th century, see Martín-Pastor, et al. (2017).
} 
construction implies the veracity of said reasoning, unlike other disciplines such as mathematics that use other demonstrative techniques.

The power of digital graphic tools, which are full of automatisms to speed up the intermediate operations and which offer, for the first time, unlimited precision in practical terms, make it possible for enhanced graphic thinking hitherto regarded as inconceivable by architects and engineers. With the help of this enhanced graphic thinking, we have addressed the reinterpretation of the inherited geometrical knowledge, in this case Archimedes' work, in order to go beyond his achievement and find a general law for one of his statements with potential applicability for the generation of architectural structures.

\section{The Classic Theorems on Intersections of Quadratic Surfaces}

The importance of the theoretical knowledge of geometrical surfaces and the properties of their intersections led to the detailed study of said surfaces in the field of applied mathematics for engineering and architecture, where quadratic surfaces held a privileged position. Quadratic surfaces are also called quadrics, of which there are 17 standard-form types, including the cone, cylinder, ellipsoid, elliptic cone, elliptic cylinder, elliptic hyperboloid, elliptic paraboloid, hyperbolic cylinder, hyperbolic paraboloid, paraboloid, sphere, and spheroid.

Since the publication of Géométrie Descriptive (1798), and with the support of algebra, a series of approximately nineteen theorems were developed concerning the intersection of quadratic surfaces. ${ }^{4}$ The best-known theorem (Fig.1), named Monge's Theorem, states:

"If two quadratic surfaces $C 1$ and $C 2$ are circumscribed about a third C3 along their contact curves $c 1$ and $c 2$, then their intersection curve decomposes into two planar curves i1 and i2, which pass through the points $A$ and $B$, which are common to the contact curves $c 1$ and c2" (Taibo-Fernández 1983:375).
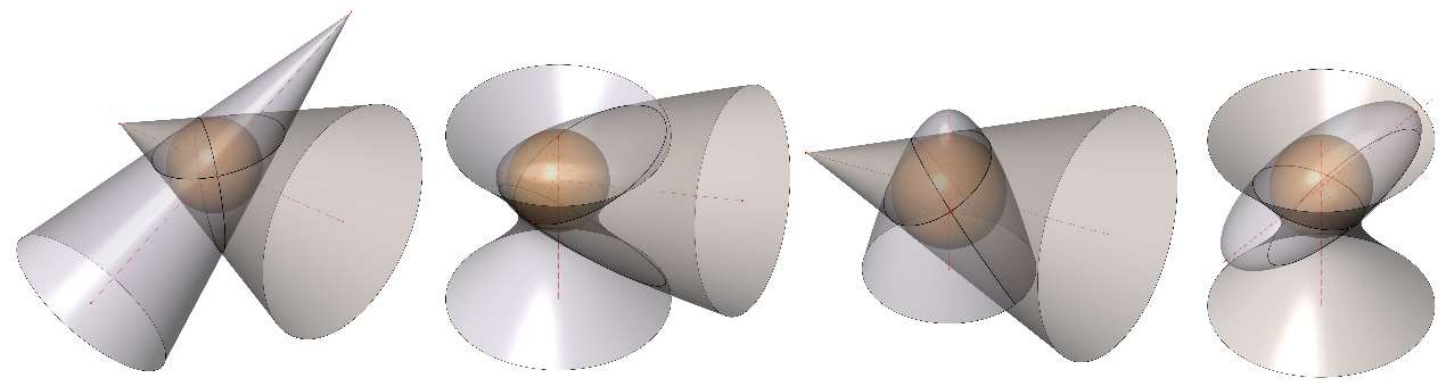

Figure 1. Examples of two quadratic surfaces circumscribed about another quadratic surface such that the intersection is composed of planar curves.

In work of a more contemporary nature by Spanish lecturers of engineering and architecture, such as Geometría Descriptiva by Taibo-Fernández (1983: 371-382), eighteen theorems are listed. In Geometría Descriptiva Superior by Izquierdo-Asensi (1985: 549-564), without actually referring to them as 'theorems', a list of nineteen statements about the properties of quadric

\footnotetext{
4 'Theorems on the intersections of quadratic surfaces - quadrics - have been studied on technical degree courses within the discipline of Descriptive Geometry. In the Spanish context, these are covered by the work of TaiboFernández (1983) and Izquierdo-Asensi (1985).
} 
intersections is provided, all formulated in a very similar way to that of Taibo's work. The statements are structured in terms of generalities, tangent quadrics, homothetic quadrics, quadrics with a general plane in common, and intersection of rotational quadrics 5 . A list of all the theorems provided by Taibo-Fernandez (1983) is listed below:

Theorem 1. The projection of the intersection between two quadratic surfaces is, in general, a curve of the fourth degree.

Theorem 2. If any two intersecting quadratic surfaces have a conic curve in common, then their intersection is completed with another conic curve. In other words, if two intersecting quadratic surfaces have a planar curve in common, then they also have another planar curve in common.

Theorem 3. If a quadratic Surface $C$ and a sphere $E$ have a circle $c 1$ in common, then whole intersection is composed of another circle c2, in addition to the first circle.

Theorem 4. The contact curve between two quadratic surfaces, which are tangent to each other, is planar.

Theorem 5. Two quadratic surfaces, tangent at any two points, intersect at two planar curves.

Theorem 6. If two quadratic surfaces $C 1$ and $C 2$ are circumscribed about a third $C 3$ along their contact curves $c 1$ and $c 2$, then their intersection curve decomposes into two planar curves i1 and i2, which pass through the points $A$ and $B$, which are common to the contact curves $c 1$ and $c 2$.

Theorem 7. Two homothetic quadratic surfaces intersect at a planar curve.

Theorem 8. When a plane sections two intersecting quadratic surfaces at homothetic curves, then any quadratic surface that passes through the intersection of the surfaces is sectioned by the same plane at a curve which is homothetic with the previous curves. Theorem 9. When two intersecting quadratic surfaces have a main plane in common, the orthogonal projection of their intersection onto said main plane is a conic curve. ${ }^{6}$ Theorem 10. When two quadratic surfaces intersect at two planar curves and have a main plane in common, the orthogonal projection of their intersection onto said main plane is reduced to two straight-line segments.

Theorem 11. If two homothetic quadratic surfaces that have the same main plane are intersected by a third quadratic surface that has the same main plane in common, then the orthogonal projections of the intersection curves onto the main plane are homothetic.

Theorem 12. If two rotational quadratic surfaces with parallel axes intersect, then the orthogonal projection of their intersection curve onto the plane containing the two axes is an arc of parabola.

Theorem 13. The intersection of two ruled quadratic surfaces with a ruling in common is composed of a straight line and a curve of the third degree.

Theorem 14. When two ruled quadratic surfaces have two converging rulings in common, the intersection is completed either with another two converging rulings or with a conic curve.

Theorem 15. When two warped ruled quadratic surfaces have two rulings from the same system in common, the rest of the intersection is composed of another two rulings from the other system.

\footnotetext{
${ }^{5}$ This last classification was used as the basis for the publication Apuntes de Geometría Descriptiva, by the Higher Technical School of Architecture of Seville, Spain, whereby seventeen of these statements were rearranged into fifteen 'Theorems on intersection of quadratic surfaces', and organised into three groups: Fundamental Theorems, Theorems on the Kind of Projected Conic Curve, and Theorems on Ruled Quadrics.

6 'Main plane' indicates a symmetry plane for the quadratic surface.
} 
Theorem 16. If two rotational quadratic surfaces with parallel axes, whose centres are on a line perpendicular to both axes, intersect, then the projection of the intersection onto a plane perpendicular to their axes is always an arc of a circle.

Theorem 17. When two intersecting quadratic surfaces have a tangent plane in common, then a cone whose apex is situated at one of the tangency points and whose directrix is the intersection curve between the two surfaces, is of the second degree. Theorem 18. Two rotational quadratic surfaces whose axes intersect, produce an intersection curve, which is orthogonally projected, onto the plane containing the axis, as an arc of hyperbola, except for the case when one of the two quadrics is an oblate ellipsoid, whereby it is projected as an arc of an ellipse. Taibo-Fernández (1983: 371382).

More recently, Professor Gentil Baldrich has added a new theorem ${ }^{7}$ to the list: The Theorem of the intruder Sphere, which states:

"A sphere, which is easily determined, can always be traced through the intersection of two rotational cones with parallel axes" (Gentil 2016:46-55).

It is difficult to elucidate the origin of each of these theorems, since a large number of contributions studying those surfaces occurred throughout the first half of the nineteenth century thanks to work by Monge, his contemporary colleagues, and disciples: Hachette (1817), Brianchon (1817), Dupin (1819, 1822), Chasles (1837, 1852, 1870), La Gournerie (1860, 1864) and Poncelet $(1862,1864)$, and many other geometers during that century.

Related to the same topic, there is also Frèzier's work (1737-1739), which can be found in Prodromes of Descriptive Geometry in the Traité de stéréotomie by Amédée François Frèzier:

"Frèzier's work, published a few years before Monge's Géométrie descriptive, summarizes the state-of-the-art of descriptive geometry in that period. Notably in the first book, Frèzier publishes an original study about the intersections between quadratic surfaces and the projective-geometrical properties of the fourth-order curves derived from them" (Salvatore 2011:271)

The so-called 'confocal' quadratic surfaces have been studied by a number of mathematicians ${ }^{8}$. However, these confocal surfaces, due to their own definition, differ from those studied in this article, since confocal quadratic surfaces share the two foci instead of only one, as is our case. Our study is limited to a group of rotational quadratic surfaces, whereas in classic studies about confocal quadratic surfaces, no distinctions are made between rotational and non-rotational surfaces.

\footnotetext{
${ }^{7}$ The Theorem of the Intruder Sphere is included for the first time in Gentil (1997:20).

8 Dinca (2014), in Thread Configurations for Ellipsoids, carries out a review of the historic contributions in confocal surfaces, and highlights the contributions by Ivory (1809), Chasles (1870), Chasles and Graves (1841), Darboux (18871896), and Staude (1883), among others. Within the group of academics that have recently studied these surfaces from different approaches to ours, work by Shene and Johnstone (1994), Miller and Goldman (1995), Lazard et al. (2006), and Bobenko et al. $(2015,2017)$ all deserve mention.
} 


\section{A theme to be analysed: On Conoids and Spheroids by Archimedes}

Closely related to the topic in hand, and going back more than two thousand two hundred years, Archimedes of Syracuse, in proposition XII of On Conoids and Spheroids, states an interesting property of rotational paraboloids ${ }^{9}$. Professor Gentil Baldrich referred to this property when he directly deduced from that proposition that "Any elliptical section of a rotational paraboloid is normally projected onto the plane perpendicular to the axis as a circumference" (Gentil 1997:26).

In Proposition XII by Archimedes ${ }^{10}$, we can read:

"Proposition XII: If through the extreme of the major axis, of the ellipse formed on a [paraboloid]..., a perpendicular line is traced until meeting the line parallel to the axis of the solid through the centre of the ellipse, the perpendicular segment traced is equal to the minor semi-axis of the aforementioned ellipse. / [...] It is thereby proved that $A K$ is equal to $P F$, in this manner." [Free translation by the authors from the Latin work by Archimedes-Maurolico (1685:256)].

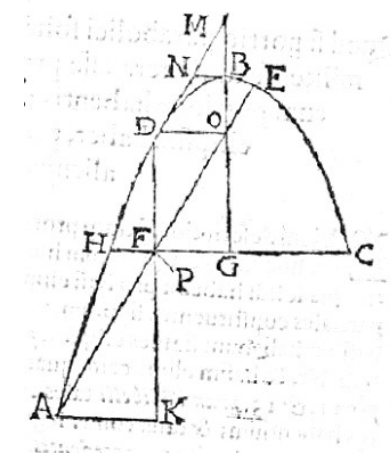

Fig. 2. Archimedes-Maurolico. De conoidibus et sphaeroidibus figuris Inventorum. Liber secundus, Proposition XII. Palermo 1685.
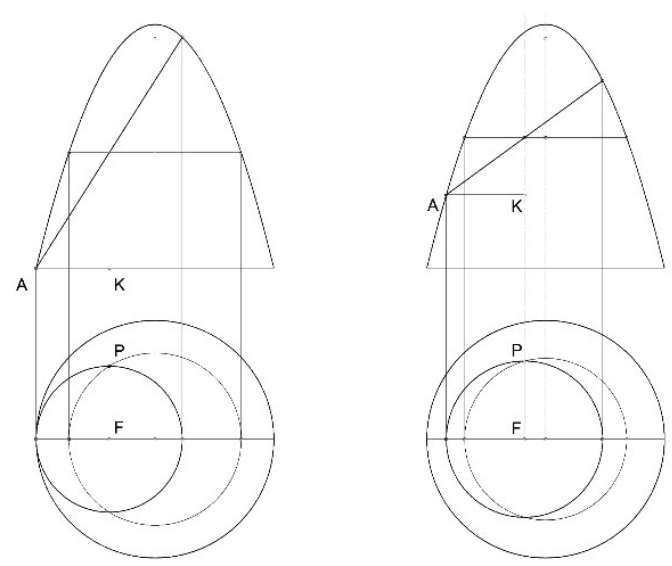

Fig. 3. Proposition XII by Archimedes.

Source: Authors' own

If the segment AK [normal projection of the semi-axis AF] is equal in length to the segment FP [semi-axis normally projected in its true size], then the ellipse AE [planar section of the paraboloid] is projected as a circumference onto the plane perpendicular to the axis of the paraboloid (Fig.2). This property holds true for any oblique planar section of the paraboloid (Fig.3).

If this property is generalised into three dimensions, and the problem is approached as a matter of quadric intersections, then the relationship between the paraboloid and the rotational cylinder generated from the aforementioned projected circle can be appreciated (Fig.4).

\footnotetext{
${ }^{9}$ The referred text by Archimedes is included in several propositions according to its various translations. The first edition of Basel includes it in Proposition XIII (Archimedes-Gechauff 1544:63). Commandino's Venetian version appears in Proposition XIII (Archimedes-Commandino 1558:35). That of Maurolico, from Palermo, is in Proposition XII (Archimedes-Maurolico 1685: 255-256). Heiberg's edition, the most rigorously commented, also includes it in Proposition XII, (Archimedes-Heiberg 1881:345).

${ }^{10}$ We have chosen Maurolico's version of 1685 since it explains the nature of the problem in a more direct way, and since it is the only translation which concludes the proposition with the equivalence in length between the two semiaxes.
} 
Fig. 4. Three-dimensional

extrapolation. Any right rotational cylinder, with its axis parallel to the axis of the paraboloid, produces a planar curve (ellipse) at its intersection with the paraboloid. Source: Authors' own.

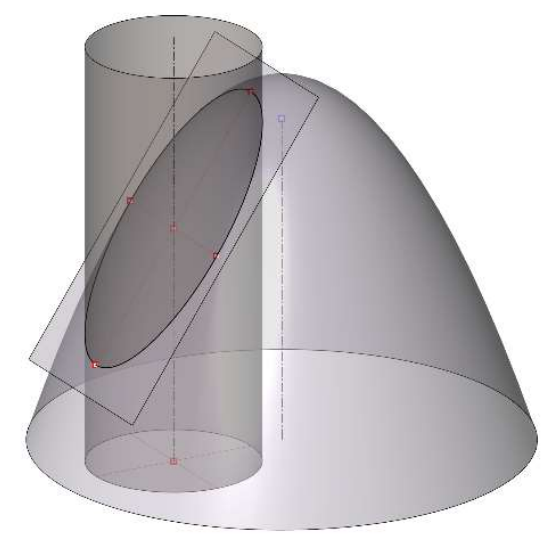

\section{Reformulating rotational quadratic surfaces from the two foci, either real or at infinity}

Henceforth, for the sake of convenience in this article, the terms quadratic surfaces or quadrics will always mean rotational quadratic surfaces.

By analysing the 'Theorems on Quadratic Surface Intersections', it can be verified that none of the classic theorems regarding quadric intersections establishes any relationship between the foci. From the position of traditional Descriptive Geometry, whose drafting tools consisted of pencil and paper, it was not practical to define a quadric in terms of its foci, and the accuracy of the drawings also failed to help in this task. The problem first posed by Archimedes, on the cylinder-paraboloid intersection, hints at how to understand the link between the foci, although for that purpose we would need to extend the affine space to understand how the foci are coupled with every quadric from the point of view of the projective space. Every quadratic surface might be understood as a 'transition surface' from a general surface with two foci (the ellipsoid) that evolves with the motion of one of these foci until infinity, or, alternatively, until both foci join at a single point.

A rotational ellipsoid has two 'real' foci, which determine the main axis of revolution. However, if the position of one of the two foci is preserved by linking it to a 'real' set point, while the other focus is taken away along the direction of the axis until it approaches infinity (let us suppose positive infinity) then the result is an ellipsoid whose shape around the set focus would be very similar to that of a paraboloid. If the mobile focus was eventually moved to infinity, then the result would be a real paraboloid (Fig.5). Therefore, the paraboloid can be understood as containing two foci, one 'real' and another 'ideal', placed at infinity, defined by the extension of one of the extremes of its main axis.

From that paraboloid (with a focus already located at positive infinity), the 'real' focus can also be moved in the opposite direction, until it approaches negative infinity, thus obtaining a rotational cylinder (Fig.6). Hence, the cylinder is a quadric with two foci at infinity: one at positive infinity and the other at negative infinity.

The remaining quadrics can be understood in the same way; a cone, for instance, stems from a rotational hyperboloid of two sheets whose foci have approached each other until their positions have coincided at the same 'real' point, which is the apex of the cone (Fig.7). Therefore, the apex of the cone can also be understood as a focal condition. In the same way, a sphere can be considered as a particular case of an ellipsoid where the two foci coincide at the same 'real' point; this case is more familiar, but retains the same nature as the previous cases. 

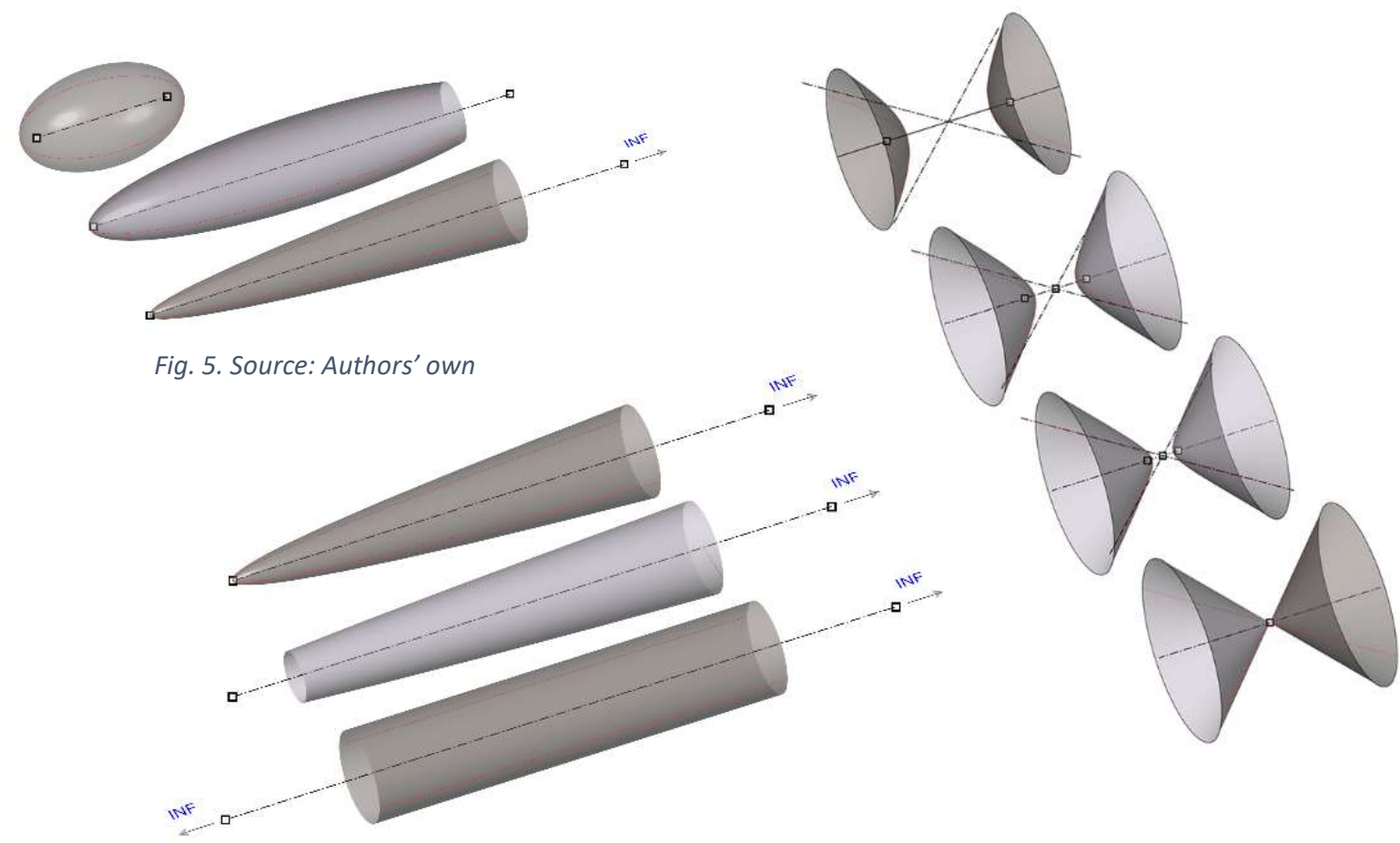

Fig. 6. Source: Authors' own

Fig. 7. Source: Authors' own

Nonetheless, according to this approach, not all quadratic surfaces can be defined by two foci. Oblate ellipsoids and one-sheeted hyperboloids are excluded. For these two surfaces the foci are not contained on the rotational axis that defines them, thus the focus produces a focal circumference when it is rotated together with the generation of the surface. For this reason, these two surfaces are excluded from this conjecture.

\section{Approach for a general hypothesis}

By applying this definition of quadrics, or quadratic surfaces, to the case of Archimedes, both paraboloids and cylinders share one of their foci at the same point of infinity. This is of great significance, as will be shown later, because this is exactly the focal relationship we were seeking. However, is the cylinder-paraboloid intersection planar since it is a special case, or it is due to the fact that it shares a focus? With this hypothesis left open, the planar intersection curve of the Archimedean interpretation could be understood as a general consequence that always occurs between two rotational quadrics as long as they share the position of one of their foci at the same point, and thus it could be the case of a general property.

Once this new hypothesis is declared, it should be verified as a general rule applicable to the remaining combinations of intersections of rotational quadratic surfaces. To this end, we have made use of Computer Aided Design tools to model the problem and verify the planarity of the intersections for all possible combinations.

Five different rotational quadratic surfaces have been considered: the ellipsoid [prolate spheroid], paraboloid, two-sheeted hyperboloid, cone, and cylinder ${ }^{11}$.

\footnotetext{
${ }^{11}$ The sphere is included as a particular case of the ellipsoid. This simplification is justified because there are no doubts about the planarity of the intersections with a sphere, since "any rotational surface intersects the surface of a sphere at one or two circumferences, as long as the axis of that surface passes through the centre of the sphere."
} 


\begin{tabular}{|l|c|c|}
\hline Rotational surface & Focus 1 & Focus 2 \\
\hline Ellipsoid [prolate spheroid] & Real & Real \\
\hline Paraboloid & Real & $\operatorname{Inf}$ \\
\hline Two-Sheeted Hyperboloid & Real & Real \\
\hline Cone & Real & \\
\hline Cylinder & $\operatorname{lnf}$ & $\operatorname{Inf}$ \\
\hline
\end{tabular}

Table 1. Summary of the rotational quadratic surfaces considered and nature of their foci.

From Table 1, the existence of 15 possible combinations can be deduced if each quadratic surface is combined with the other surfaces and with itself. In addition, there is a second combination of the paraboloid with itself, in order to take into account the coincidence at both the real focus and the ideal focus. Therefore, there are a total number of 16 combinations. In this way, not only do the combinations take into account the kind of surface involved, but also the nature of their foci (real or ideal).

From the 16 combinations, 11 produce conic curves at their intersections, 2 produce straight lines degenerated from conic curves, and 3 produce impossible combinations, since it was impossible to make a real focus and an ideal focus coincide.

For each of the 11 combinations that produce conical curves, according to the graphic nature of our methodological approach, it is necessary to verify the condition of planarity. To this end, it is necessary to consider different cases with dimensional and positional variations within each combination. From the infinite number of possible cases, a considerable number of samples have been selected. Some of these samples have been produced by randomly changing dimensional and positional values of each surface. For instance, for a cone, the conical angle and the axis direction are random. In addition, deterministic combinations have been employed to ensure that all possible particular situations are handled. For example, when the intersection between two hyperboloids sharing the position of a focus was studied, different cases were produced depending on the number of the sheets of the hyperboloids involved in the intersection.

For each and every combination and case, it has been empirically tested that when the quadrics share the position of one of their foci, then the intersections obtained are planar curves (conics), thereby validating the initial hypothesis to finally state our conjecture. 


\section{Verification}

Combination 1. Ellipsoid-Ellipsoid intersection sharing the position of a focus at the same 'real' point (Fig.8).
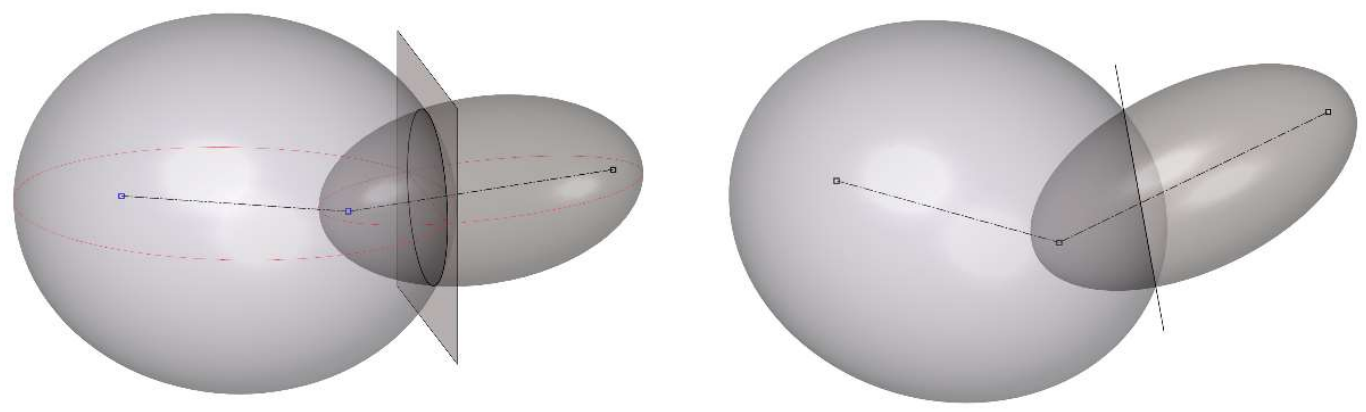

Fig. 8. Ellipsoid-Ellipsoid intersection. The intersection between two ellipsoids sharing the position of a focus at the same "real" point is a planar curve, and, in general, an ellipse. This supports the initial hypothesis. Source: Authors' own

Combination 2. Ellipsoid-Paraboloid intersection sharing the position of a focus at the same 'real' point (Fig.9).
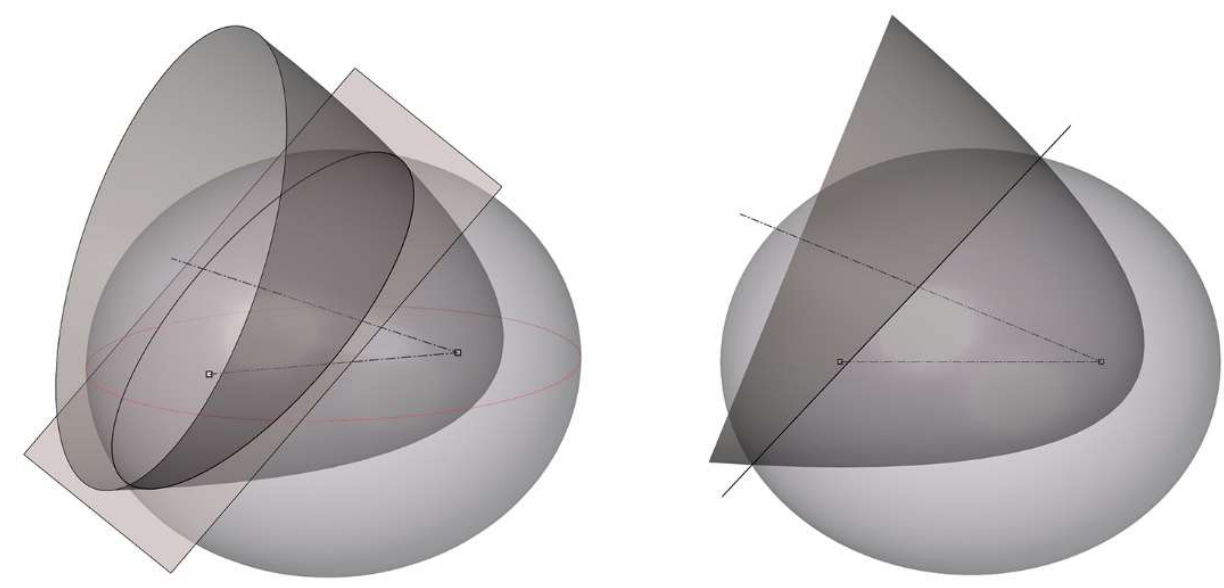

Fig. 9. Ellipsoid-Paraboloid intersection. The intersection between an ellipsoid and a paraboloid sharing the position of a focus at the same "real" point is a planar curve, and, in general, an ellipse. This supports the initial hypothesis. Source: Authors' own 
Combination 3. Ellipsoid-Hyperboloid intersection sharing the position of a focus at the same 'real' point (Fig.10).
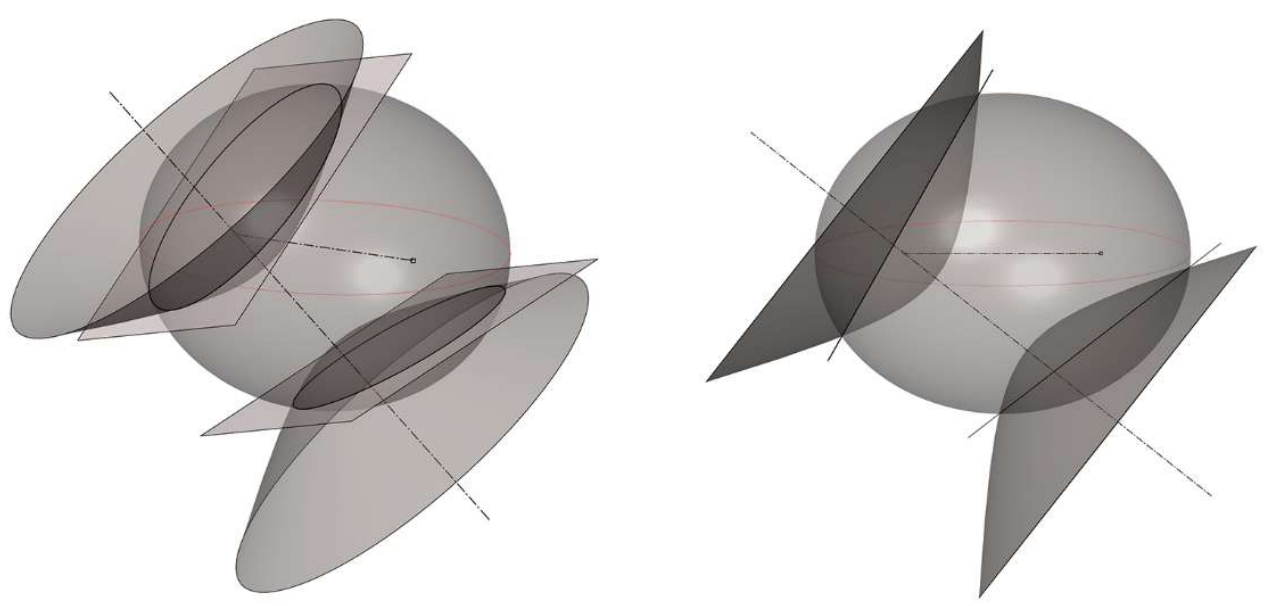

Fig. 10. Ellipsoid-Hyperboloid intersection. The intersection between an ellipsoid and a two-sheeted hyperboloid sharing the position of a focus at the same "real" point is two planar curves, and, in general, two ellipses. This supports the initial hypothesis. Source: Authors' own

Combination 4. Ellipsoid-Cone intersection sharing the position of a focus and the apex at the same 'real' point (Fig.11).
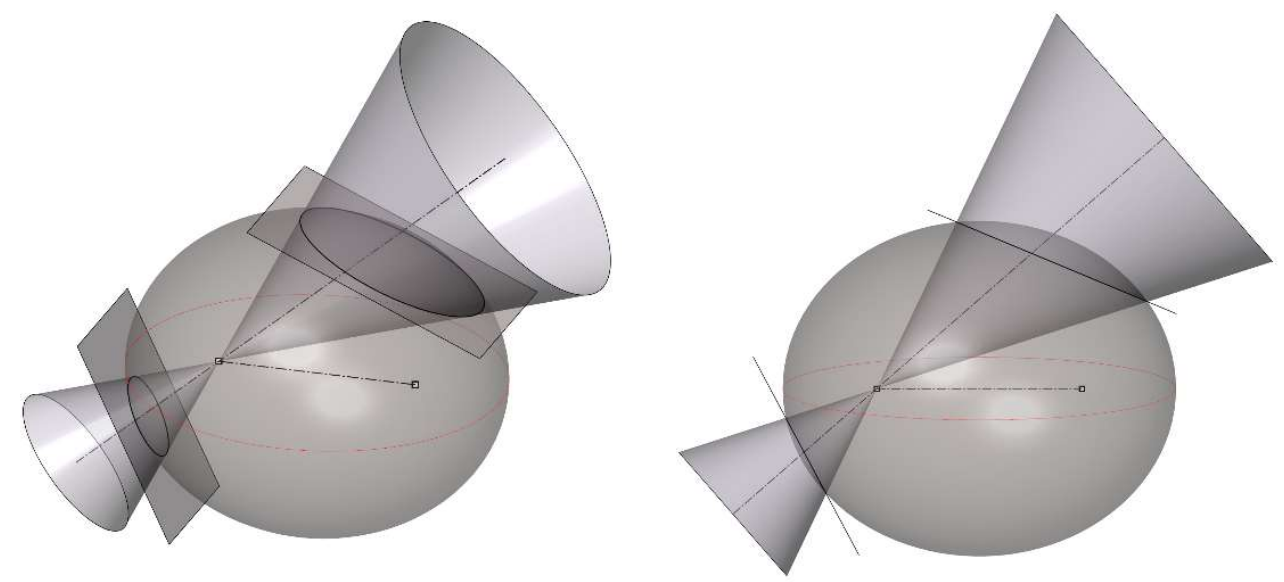

Fig. 11. Ellipsoid-Cone intersection. The intersection between an ellipsoid and a cone sharing the position of a focus (as the apex in the cone) at the same "real" point is two planar curves, and, in general, two ellipses, each of which are on a different sheet of the cone. This supports the initial hypothesis. Source: Authors' own 
Combination 5. Paraboloid-Cylinder intersection sharing the position of a focus at the same 'ideal' point (Fig.12). The two surfaces have parallel axes that meet at a point of infinity.
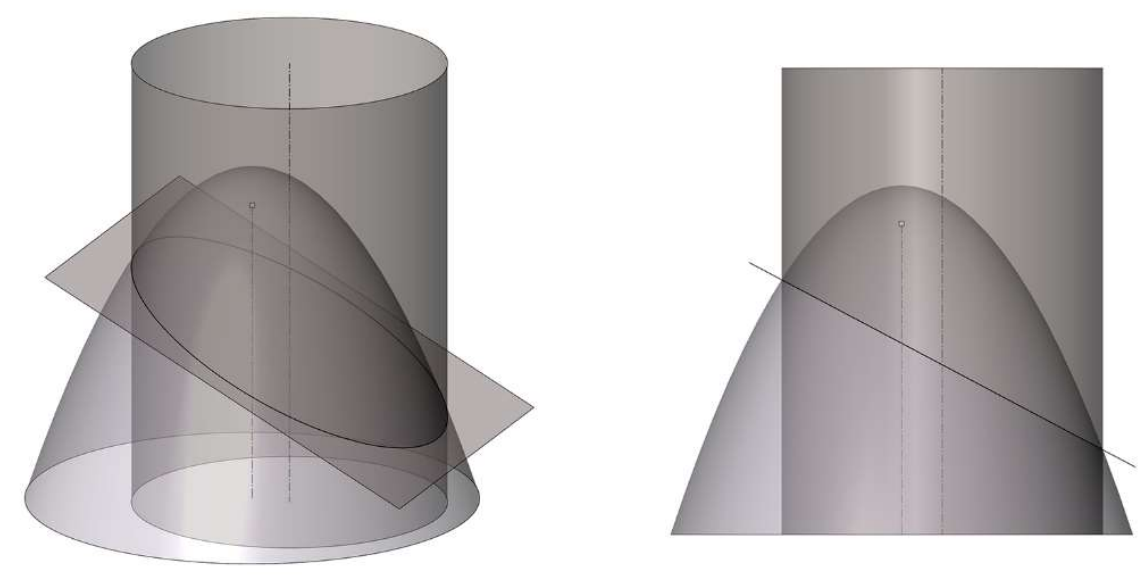

Fig. 12. Paraboloid-Cylinder intersection. This is exactly the case analysed by Archimedes. As shown in the figure, the intersection between a paraboloid and a cylinder sharing the position of a focus at the same "ideal" point (the two surfaces have parallel axes that meet at a point of infinity) is a planar curve, and, in general, an ellipse. This supports the initial hypothesis. Source: Authors' own

Combination 6. Paraboloid-Paraboloid intersection sharing the position of a focus at the same 'ideal' point (Fig.13). The two surfaces have parallel axes that meet at a point of infinity.
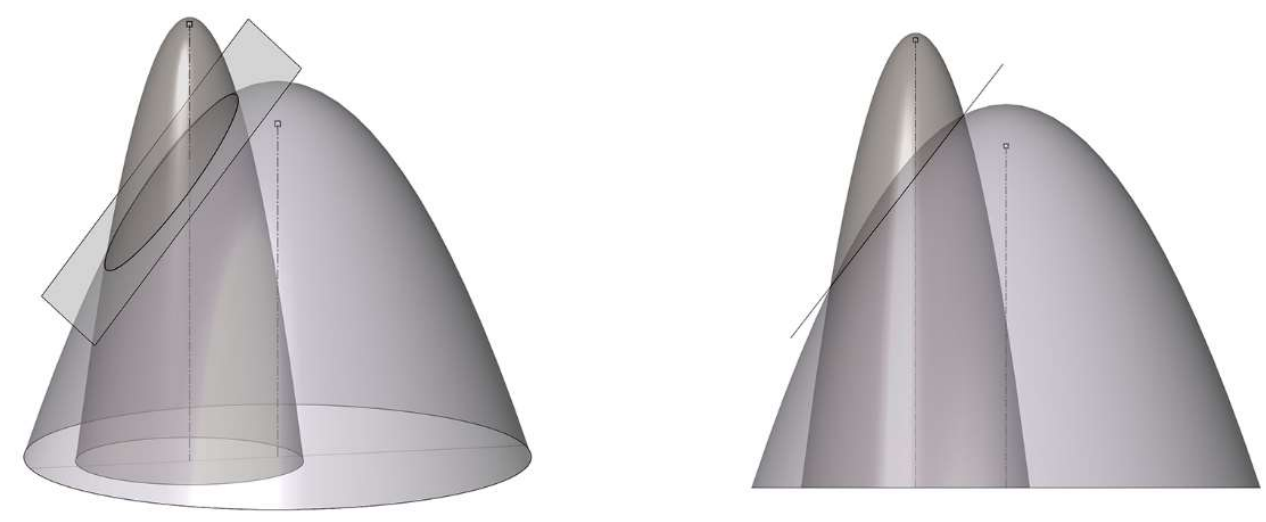

Fig. 13. Paraboloid-Paraboloid intersection. The intersection between two paraboloids sharing the position of a focus at the same "ideal" point (the two surfaces have parallel axes that meet at a point of infinity) is a planar curve, and, in general, an ellipse, which would degenerate into a parabola if both paraboloids had the same scale. This supports the initial hypothesis. Source: Authors' own 
Combination 7. Paraboloid-Paraboloid intersection sharing the position of a focus at the same 'real' point (Fig.14).

Fig. 14. Paraboloid-Paraboloid intersection. The intersection between two paraboloids sharing the position of a focus at the same "real" point is a planar curve, and, in general, an ellipse. This supports the initial hypothesis. Source: Authors' own
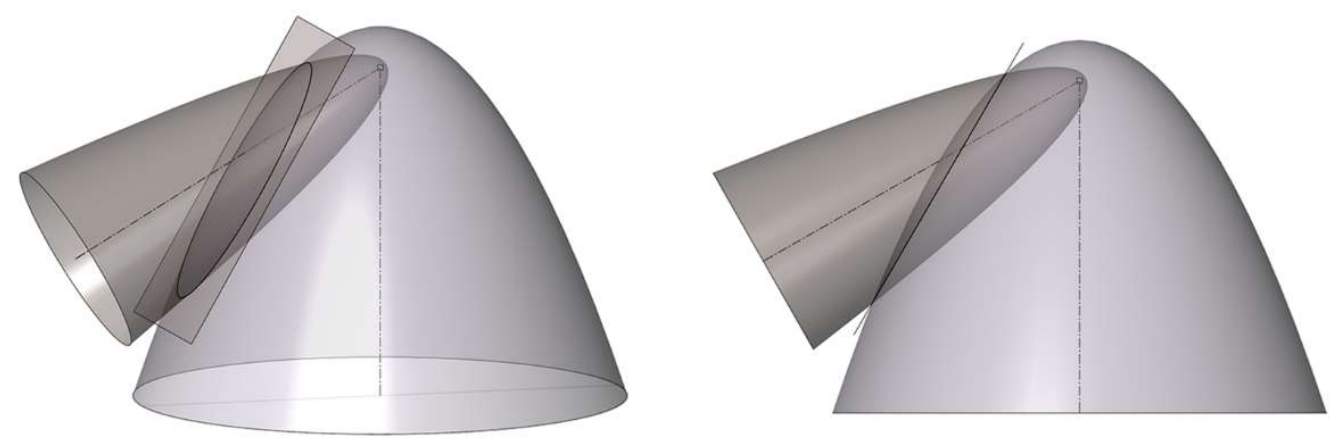

Combination 8. Paraboloid-(Two-sheeted) hyperboloid intersection sharing the position of a focus at the same 'real' point (Fig.15).
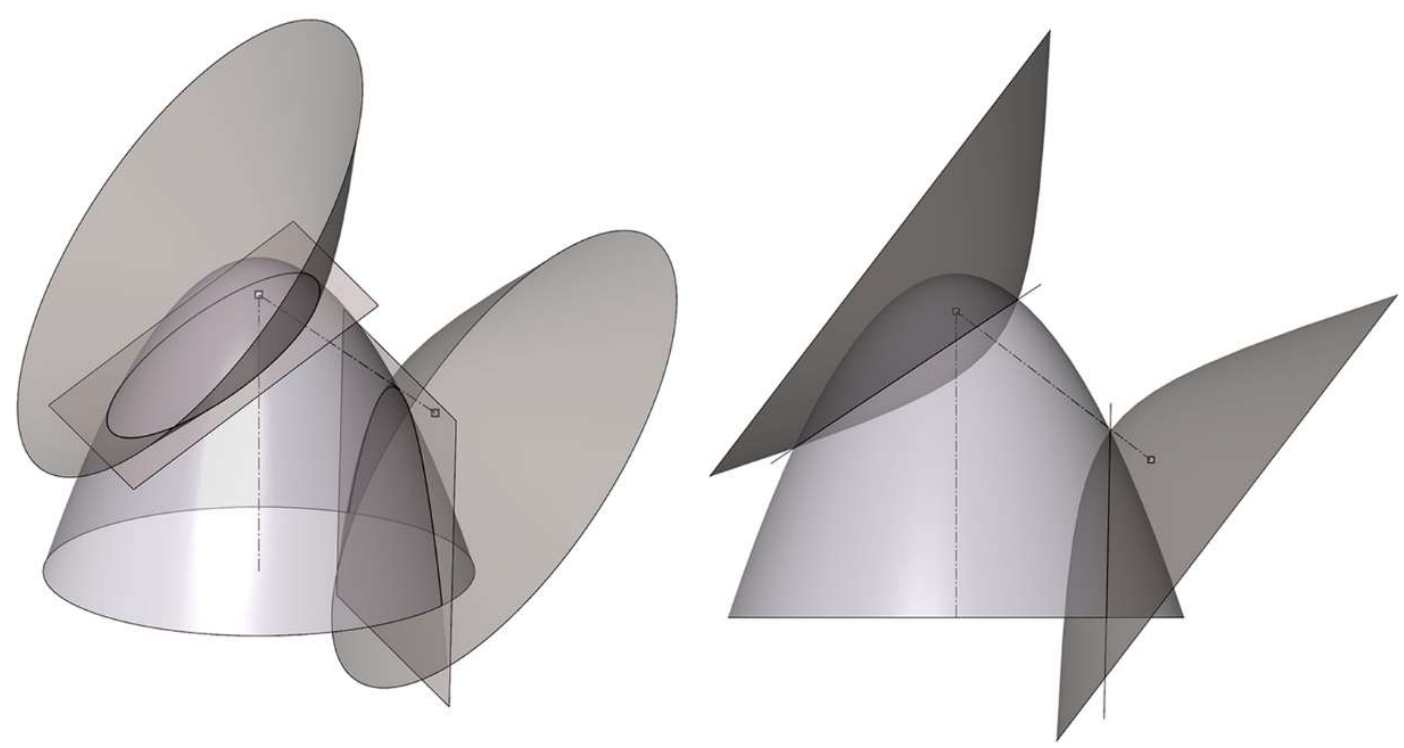

Fig. 15. Paraboloid-Hyperboloid intersection. The intersection between a paraboloid and a hyperboloid sharing the position of a focus at the same "real" point is two planar curves, and, in general, two ellipses, which would degenerate into a parabola if the plane containing these curves were parallel to the axis of the paraboloid. This supports the initial hypothesis. Source: Authors' own 
Combination 9. Paraboloid-Cone intersection sharing the position of a focus and the apex at the same 'real' point (Fig.16).
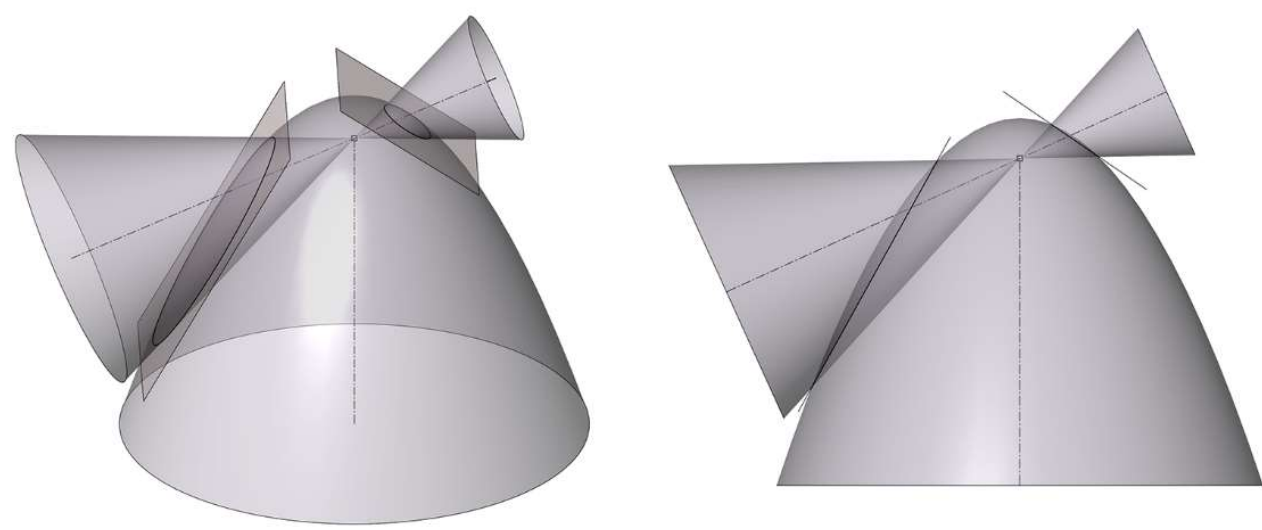

Fig. 16. Paraboloid-Cone intersection. The intersection between a paraboloid and a cone sharing the position of a focus (as the apex in the cone) at the same "real" point is two planar curves, and, in general, two ellipses, each of which are on a different sheet of the cone. This supports the initial hypothesis. Source: Authors' own

Combination 10. Intersection of two two-sheeted hyperboloids sharing the position of a focus at the same 'real' point (Fig.17).

Fig. 17. Hyperboloid-Hyperboloid intersection. The intersection between two hyperboloids sharing the position of a focus at the same "real" point is a planar curve, and, in general, a hyperbola. This supports the initial hypothesis. Source: Authors' own
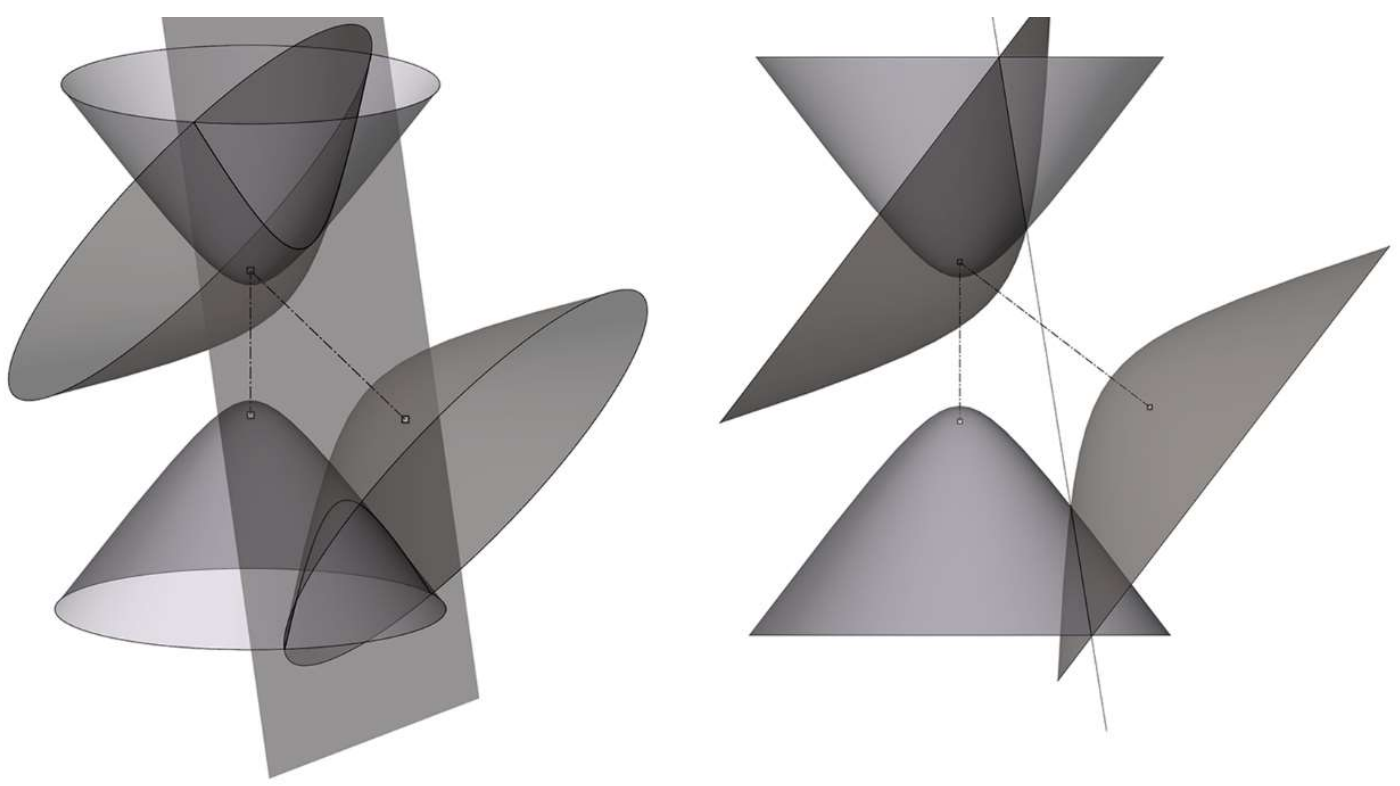
Combination 11. Two-sheeted hyperboloid-Cone intersection sharing the position of a focus and the apex at the same 'real' point (Fig.18).
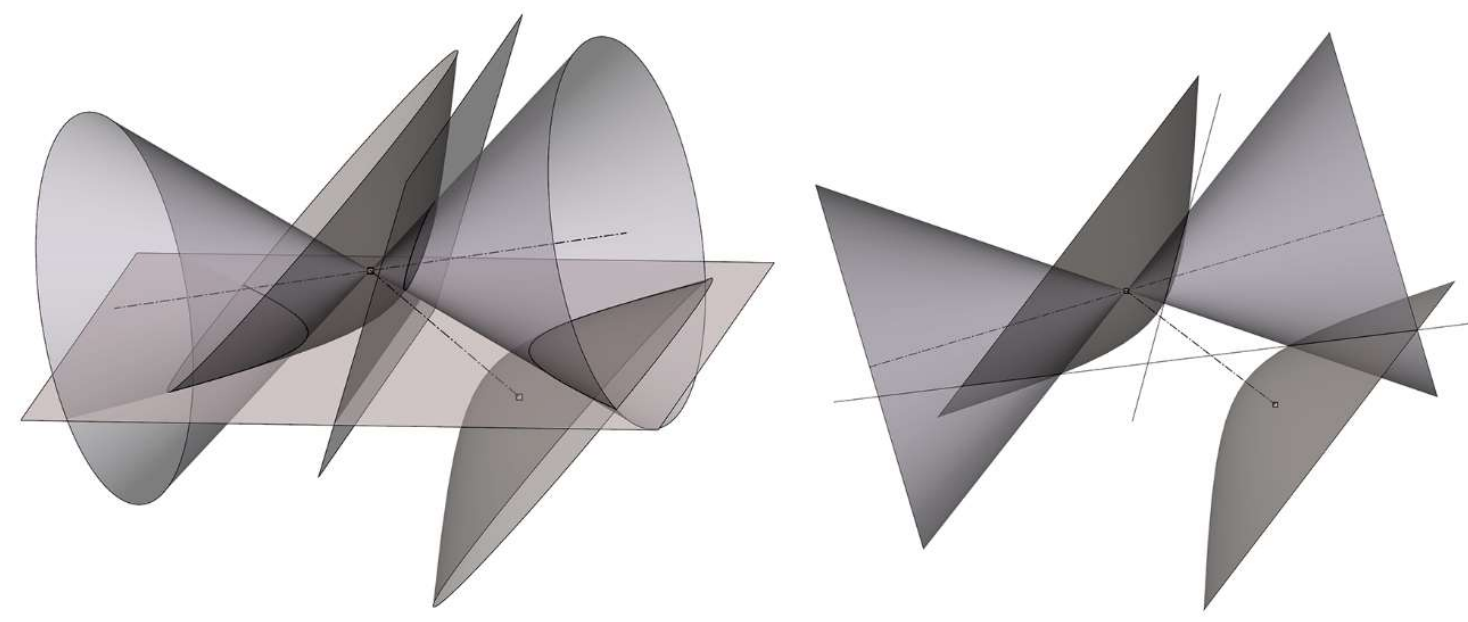

Fig. 18. Hyperboloid-Cone intersection. The intersection between a hyperboloid and a cone sharing the position of a focus (as the apex in the cone) at the same "real" point is two planar curves, and, in general, a hyperbola (with the two branches) and an ellipse. This supports the initial hypothesis. Source: Authors' own

Note 1: In the Combination of the intersection of two cylinders with parallel axes, the intersection is a pair of parallel straight lines: common generatrices of both surfaces which can be considered as the degenerated case of a conic.

Note 2: In the Combination of the intersection of two cones sharing the same apex, the intersection is a pair of straight lines: common generatrices of both surfaces which can be considered as the degenerated case of a conic.

Note 3: The Ellipsoid-Cylinder combination. In accordance with the general rule stated, the ellipsoid has the two foci within the affine space, whereas the cylinder has the two foci at infinity, and therefore it is impossible for these two surfaces to share any of the foci at the same position.

Note 4: The Hyperboloid-Cylinder combination. In accordance with the general rule stated, the hyperboloid has the two foci within the affine space whereas the cylinder has the two foci at infinity, thus it is impossible for these two surfaces to share any of the foci at the same position.

Note 5: The Cone-Cylinder combination. In accordance with the general rule stated, the cone has the two foci coincident at its apex and the cylinder has the two foci at infinity, thus it is impossible for these two surfaces to share any of the foci at the same position. 


\section{Applications in architecture and engineering}

The usefulness of the initial Archimedean property has already been proved for the generation of algorithms in architectural computational design. The aforementioned projective interpretation (Gentil 1997:26) can be used to produce planar compositions of circles, or circular arcs, located on a base plane perpendicular to the axis of a rotational parabolic dome. Therefore, the projection of these compositions onto the parabolic surface results in planar ellipsis, or elliptical arcs, which provides an appropriate geometric framework for both discretising the surface with planar elements and translating the projected composition into material elements for architectural purposes.

This is the case of the Archimedean Pavilion, a full-scale prototype developed with algorithms stemming from this property. The starting point is a composition of an architectural space composed of various inclined rotational parabolic domes. Each dome is discretised by using a composition based on the circle packing of the boundary of the parabolic fraction projected onto the base plane. Once this composition is projected onto the dome, the parabolic surface is substituted with the set of ellipses obtained. Finally, these ellipses are materialised by three fractions of conical surfaces that provide sufficient rigidity to form a self-supporting structural system applicable to any parabolic dome through the use of digital fabrication techniques. This is an efficient lightweight system, which, combined with the structural strengths of the general shape of rotational paraboloids with vertical axes, is especially appropriate for the materialisation of wide-span roofs, and includes the possibilities of glazing or panelling, as can be consulted in (Narvaez-Rodriguez and Barrera-Vera, 2016), (Fig.19).
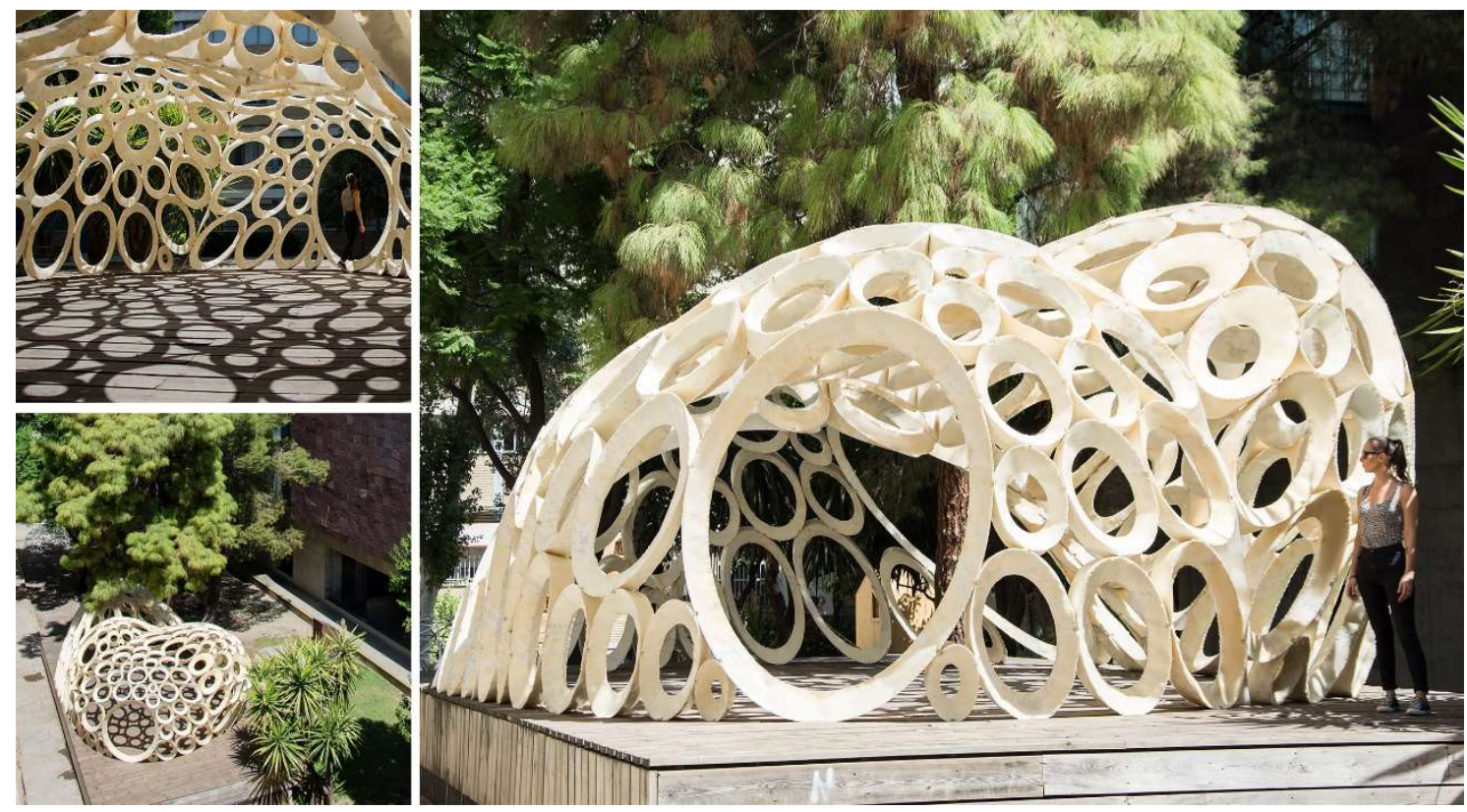

Fig. 19. Photographs of the Archimedean Pavilion (by Roberto Narvaez-Rodriguez), 2016, composed of four inclined rotational paraboloids which were discretised with an algorithm based on the initial property stated by Archimedes. Source: Authors' own

The conjecture stated in this article opens more possibilities for the materialisation of not only parabolic domes, but also for domes or architectural surfaces based on the use of any rotational quadratic surface as described previously, since the planarity achieved for the intersection 
curves is always a property that facilitates its translation into constructive components through digital fabrication techniques. On the one hand, the new projective properties stemming from the combinations of cones and cylinders with the other quadratic surfaces can be employed to generate planar intersection curves that populate and discretise these surfaces. On the other hand, new algorithms can also be developed for the discretisation and panelling of quadratic surfaces based on the variety of possibilities that the intersection of these rotational quadrics provides.

A simple example, one of the wide range of potential applications, is given by the discretisation of a rotational ellipsoid shown in (Fig.20). Using the conjecture stated in this document and with the aim of populating the elliptical surface with planar curves [ellipses in this case], the following operations, generally described, have been implemented in an algorithm:

- Draw the target ellipsoid and identify the two foci.

- Draw a sphere whose centre coincides with one of the foci of the ellipsoid and with an arbitrary radius.

- Draw any composition of circles on the surface of the sphere. In this case, circle packing, combined with some special circles [not tangent to their neighbours] to fill gaps, has been used.

- Project the composition of circles from the centre of the sphere, one of the ellipsoid's foci, onto the ellipsoid's surface. This operation implies two important conditions. The first condition is that the projection of every circle can be understood as the intersection of a rotational cone with the ellipsoid. This rotational cone has its apex in the centre of the sphere, one of the ellipsoid's foci, and its base is the circle on the sphere's surface. This ensures that the cones remain rotational and hold the position of their apex [degenerated foci] at the ellipsoid's focus, thus accomplishing the conditions of the conjecture. The second condition is derived from the conjecture itself, in that it requires the planarity of all intersection curves obtained on the ellipsoid's surface. These planar curves populating the elliptical surface can be used as a geometric structure to materialise a construction system, such as that performed in the Archimedean Pavilion.
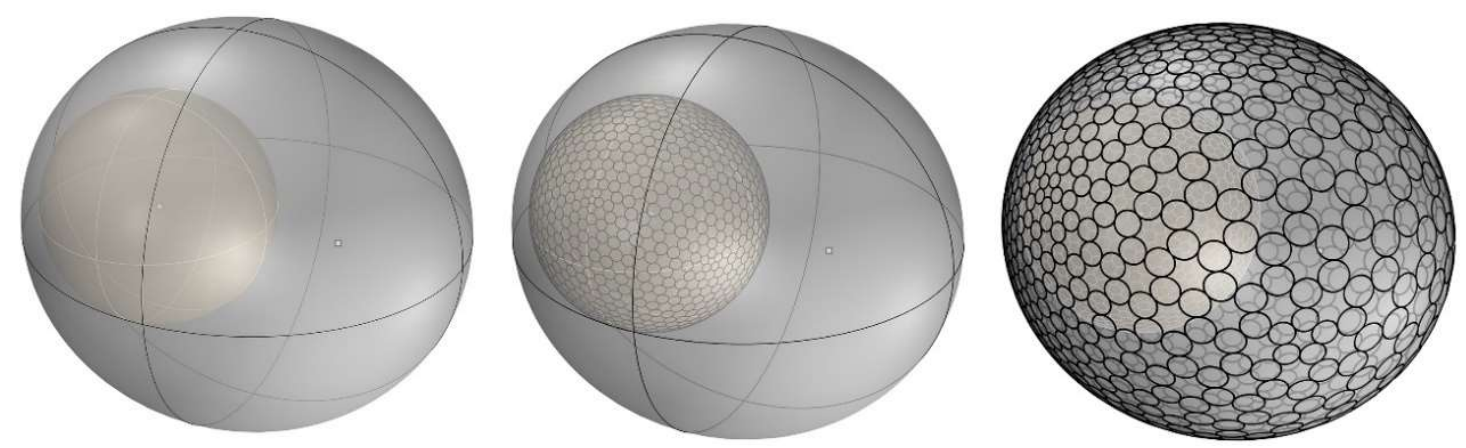

Fig. 20. Discretisation of an ellipsoid using an algorithm based on the conjecture. Left: Target ellipsoid and sphere whose centre coincides with one the ellipsoid's foci. Middle: composition of circles on the sphere to define the rotational cones. Right: Planar curves, ellipses, obtained from the Intersection of the cones with the ellipsoid's surface. Source: Authors' own 


\section{Conclusion}

After carrying out the relevant verifications, all the indications are that our approach, deduced from the particular case of Archimedes, holds true for all combinations and cases. This group also includes the combination of a rotational cylinder and paraboloid, both considered with one of their foci at infinity and that of a rotational cone and sphere, both considered with the two foci coinciding at a single point. Therefore, a new general conjecture on the intersection of rotational quadrics can be stated:

"If two rotational quadratic surfaces share the position of one of their two foci, the intersection curves between the two surfaces are always planar".

The use of CAD systems combined with the algorithmic design possibilities provide a powerful tool, not only for the generation of new geometries, but also for the reinterpretation of classic geometric properties and concepts. This approach can provide both new insights and discoveries which have never been considered with classic tools, and new geometric structures for known surfaces whose widespread applicability in architecture still provides appropriate solutions.

\section{Acknowledgements}

We would like to thank prof. Dr. José María Gentil Baldrich for introducing us to this line of research. We would like to thank prof. Daniel Hernández Macías for his participation in this research, without which the results achieved would not have been possible. We also want to emphasize his contribution as a co-author of the conjecture. Our thanks also go to prof. Dr. María-José Chávez for her corrections and mathematical advice.

We would like to thank the sponsors of the Archimedean Pavilion: University of Seville, through ETSIE, Department of Graphic Engineering and Vicerrectorado de Estudiantes, and Dow Building Solutions [a division of The Dow Chemical Company]. Thanks also to Shuming Wang Zhu for his collaboration and to the group of students, lecturers, FabLab Sevilla, and Ehcofab for their support and collaboration throughout the development, fabrication and assembly of the Archimedean Pavilion (http://archimedeanpavilion.blogspot.com.es). 


\section{References}

Arquímedes-Gechauff, Thomas (Alias Venatorius). 1544. Archimedis Syracusani ... opera, quae quidem extant, omnia, multis iam seculis desiderata, atque a quam paucissimis hactenus visa, nuncque primum \& Graece \& Latine in lucem edita, Basel: Herwagen, Johannes d.Ä. http://dx.doi.org/10.3931/e-rara-8997

Arquímedes-Commandino. 1558. Archimedis opera non nulla a Federico Commandino. Venice: P. Manutium. Aldus. [Two parts, the second: Comenterii in opera non nulla Archimedis]. http://gallica.bnf.fr/ark:/12148/bpt6k58156t

Arquimedes-Maurolico, Francesco. 1685. Monumenta omnia mathematica, quae extant, quorumque catalogum inversa pagina demonstrat: opus praeclarissimum, non prius typis commissum, a matheseos vero studiosis enixe desideratum, tandemque e fulgine temporum accurate excussum / ex traditione Francisci Maurolici: Panormi [Palermo] : Apud Cyllenium Hesperium, 1685. [De Conoidibus et Sphaeroibidus in two books: 1ํ pp. 226-246; 20 pp. 247275]. http://dx.doi.org/10.3931/e-rara-12132

Arquímedes-Heiberg, Johan Ludvig. 1880. Archimedis opera omnia cum commentariis Eutocii. Vol 1. Lipsiae [Leipzig]: In aedibus B. G. Teubner. http://archive.org/details/archimedisoperao01arch

Bobenko, A. I., Schief, W. K., Suris, Y. B. and Techter, J. 2015. On a discretization of confocal quadrics. I. An integrable systems approach. Journal of Integrable Systems, 1(1), 1-34. https://doi.org/10.1093/integr/xyw005

-. 2017. On a discretization of confocal quadrics. II. A geometric approach to general parametrizations. arXiv:1708.06800 [math.DG]

Brianchon, Charles-Julie. 1817. Mémoire sur les lignes du second ordre. Paris: Bachelier.

Chasles, Michel. 1837. Aperçu Historique sur l'origine et le développement des méthodes en géométrie, particulièrement de celles qui se rapportent a la géométrie moderne, Bruxelles: $\mathrm{M}$. Hayez.

—. 1852. Traité de géométrie supérieure, París: Bachelier.

-. 1870. Rapport sur les progrès de la géométrie, par M. Chasles, Paris: Impr. Nationale.

Chasles, Michel and Graves, Charles. 1841. Two Geometrical Memoirs on the General Properties of Cones of the Second Degree. (Trad. additions and appendix by Graves, C.). Dublin: Graisberry and Gill.

Darboux, Gaston. 1896. Leçons sur la théorie génerale des surfaces et les applications géométriques du calcul infinitésimal, Vol 2-3, Paris: Gauthier-Villars.

Dinca, lon. 2014. Thread configurations for ellipsoids. Rev. Roumaine Math. Pures Appl., (3), 371-398.

Dupin, Charles. 1819. Essai historique sur les services et les travauxs cientifiques de Gaspard Monge, par M. Ch. Dupin, Paris: Bachelier.

-. 1822. Applications de géométrie et de méchanique à la marine, aux ponts-et-chaussées, etc., pour faire suite aux "Développements de géométrie" par Charles Dupin,..., Paris: Bachelier. 
Frèzier, Amédée François. 1737-1739. La théorie et la pratique de la coupe de pierres et de bois pour la construction des voûtes, et autre parties des bâtiments civils et militaire, ou traité de stéréotomie à l'usage de l'architecture. Strasbourg-Paris: Jean Daniel Doulsseker-L. H. Guerin.

Gentil Baldrich, José María. 1997. Sobre la intersección de las cuádricas de revolución de ejes paralelos. Sevilla: Departamento de Expresión Gráfica Arquitectónica.

—. 2016. Teorema de la esfera intrusa. In: ACCA 015. Sevilla/Málaga: dEGA/RU Books.

Hachette, Jean Nicolas Pierre. 1817. Éléments de Géométrie à trois dimensions (partie synthétique), de M. Hachette. Paris: L'Auteur.

Izquierdo-Asensi, Fernando. 1985. Geometría Descriptiva Superior y Aplicada. Madrid: Dossat [10 edición 1978].

Ivory, James. 1809. On the attraction of homogeneous ellipsoids. Phil. Trans. Royal Soc. London, 99, 345-372.

Lazard, S., Peñaranda, L. M. and Petitjean, S. 2006. Intersecting Quadrics : An Efficient and Exact Implementation. Computational Geometry, 35, 74-99.

https://doi.org/10.1016/j.comgeo.2005.10.004

Martín-Pastor, A. and Granado-Castro, G. (2017). Some controversies on representation of the solar shadow in 17th century. The manuscript 'Artes Excelençias de la Perspectiba' in context. Disegnare idee immagini, 54, 12-23.

Miller, J. R. and Goldman, R. N. 1995. Geometric algorithms for detecting and calculating all conic sections in the intersection of any two natural quadric surfaces. Graphical Models and Image Processing, 57(1), 55-66. https://doi.org/https://doi.org/10.1006/gmip.1995.1006

Monge, Gaspard. 1798. Géométrie Descriptive - Lecons données aux Écoles normales, I'An 3 de la République; Par Gaspard Monge. Paris: Impr. de Baudouin.

-. 1809. Application de l'analyse à la géométrie, à l'usage de l'Ecole impériale polytechnique; par M. Monge. Paris: Ve Bernard [1a ed. in-fol, 1795, Feuilles d'Analyses appliquée à la Géométrie, Ecole polytechnique]

Narvaez-Rodriguez, R. and Barrera-Vera, J. A. (2016). Lightweight Conical Components for Rotational Parabolic Domes: Geometric Definition, Structural Behaviour, Optimisation and Digital Fabrication. In S. Adriaenssens, F. Gramazio, M. Kholer, A. Menges, \& M. Pauly (Eds.), Advances in Architectural Geometry 2016 pp. 378-397. Zurich: vdf Hochschulverlag AG an der ETH Zürich. https://doi.org/10.3218/3778-4_25

Poncelet, Jean-Victor. 1862-1864. Applications d'analyse et de géométrie qui ont servi de principal fondement au Traité des propriétés projectives des figures / par J.-V. Poncelet; et accompagnés de divers autres écrits... par MM. Mannheim et Moutard,... Paris: MalletBachelier.

Raynaud, Dominique. 2018. Sociologie des controverses scientifiques. Paris: Édition Matériologiques [1st edition 2003].

Salvatore, Marta. 2011. Prodromes of Descriptive Geometry in the Traité de stéréotomie by Amédée François Frézier. Nexus Network Journal, 13(3), 671-699. https://doi.org/10.1007/s00004-011-0086-0 
Shene, C. K. and Johnstone, J. K. 1994. On the Lower Degree Intersections of Two Natural Quadrics. ACM Trans. Graph., 13(4), 400-424. https://doi.org/10.1145/195826.197316

Staude, Otto. 1883. Geometrische Deutung der Additionstheoreme der hyperelliptischen Integrale und Functionen. Mathematische Annalen, XXII (1), 1-69, 146-176.

Taibo-Fernández. 1983. A. Geometría Descriptiva y sus aplicaciones. Tomo II. Madrid: Tébar Flores. 